\title{
Características clínico-patológicas del carcinoma de células renales detectado incidentalmente mediante estudios radiológicos
}

\author{
Bulnes Vázquez V*, Álvarez-Múgica M**, Fernández Gómez JM**, Nava Tomás E*, \\ Jalón Monzón A**, Meilán Martínez Á*.
}

*Servicio de Radiodiagnóstico 1. **Servicio de Urología 1. Hospital Universitario Central de Asturias.

Actas Urol Esp. 2008;32(10):976-984

\section{RESUMEN}

CARACTERÍSTICAS CLÍNICO-PATOLÓGICAS DEL CARCINOMA DE CÉLULAS RENALES DETECTADO INCIDENTALMENTE MEDIANTE ESTUDIOS RADIOLÓGICOS

Objetivos: Evaluar el motivo de diagnóstico del carcinoma renal y su variación a lo largo de los años en los Servicios de Radiodiagnóstico 1 y Urología 1 del Hospital Universitario Central de Asturias (HUCA), comparando los periodos 1970-1995 y 1996-2005 y determinar si el hallazgo incidental puede explicar de forma independiente el pronóstico de estos tumores o es dependiente de sus características clínico-patológicas.

Material y métodos: Se llevó a cabo una revisión exhaustiva de las historias clínicas, de manera retrospectiva, de 373 carcinomas de células renales sometidos a resección quirúrgica entre los años 1970 y 2005, recogiéndose unos datos según un protocolo establecido.

Resultados: De los 373 tumores de nuestra serie, 146 (39,1\%) fueron diagnosticados según nuestros criterios de forma incidental, mientras que $227(60,9 \%)$ lo fueron por presentar clínica sospechosa de presencia de un tumor. Cuando realizamos el recuento distribuyendo estos pacientes en dos grupos, por un lado los diagnosticados entre los años 1970-1995, y por otro lado los diagnosticados entre 1996-2005, observamos un incremento significativo en el número de tumores diagnosticados de forma incidental en el segundo periodo. Encontramos como factores independientes en la supervivencia cáncer específica la presencia de metástasis, la invasión microvascular de la pieza quirúrgica y el tratamiento de la recidiva (quirúrgico vs otros), mientras que los factores independientes de la recidiva resultaron ser el grado nuclear, la presencia de adenopatías y el estadio T3, por tanto la presencia de clínica no fue un factor independiente de recidiva ni de supervivencia cáncer específico.

Conclusiones: Clásicamente el carcinoma de células renales se diagnosticaba cuando presentaba síntomas locales (la hematuria y el dolor lumbar, eran los más frecuentes), síntomas derivados de las metástasis o por la expresión de un síndrome paraneoplásico. Desde la generalización de la ecografía y la tomografía computerizada (TC) en el estudio de la patología abdominal ha aumentado de forma espectacular el descubrimiento de adenocarcinomas renales asintomáticos. Cabe destacar, en nuestro estudio, que a pesar de que los pacientes diagnosticados con tumores incidentales presentaron un mayor tiempo libre de enfermedad y de supervivencia sin embargo, la presencia de clínica no fue un factor independiente ni de recidiva ni de supervivencia cáncer específico.

Palabras clave: Carcinoma de células renales. Ecografia. Escáner.

\section{ABSTRACT}

CLINICOPATHOLOGIC FEATURES OF RENAL CELL CARCINOMA INCIDENTALLY DETECTED THROUGH RADIOLOGICAL STUDIES

Objectives: To evaluate the reason for diagnosis of renal cell carcinoma and its variation over the years in Radiology 1 and Urology 1 departments of the Asturias Central Universitary Hospital (HUCA) comparing periods 1970-1995 and 1996-2005 and determine whether incidental findings may explain in an independent the prognosis of these tumors or it is dependent on its clinicopathologic characteristics.

Material and methods: We conducted retrospectively, an exhaustive review of medical records of 373 renal cell carcinoma patients that underwent surgical resection during the period 1970-2005, collecting data according to a set protocol.

Results: Of the 373 tumors in our series, $146(39,1 \%)$ were diagnosed according to our criteria incidentally, while 227 (60,9\%) were diagnosed due to clinical suspicion of a tumor presence. We observed a significant increase in the number of tumors incidentally diagnosed in the second period, when we performed the counting distributing these patients into two groups, firstly those diagnosed between the years 1970-1995 and, secondly, those diagnosed between 1996-2005. We found metastasis, microvascular invasion of surgical piece and treatment of the recurrence as independent factors for specific cancer survival, while nuclear grade, the presence of lymphadenopathies and T3 stage were found as independent factors for recurrence.

Conclusions: Classically renal cell carcinoma was diagnosed when local symptoms were present (hematuria and back pain, were the most common), symptoms associated with metastasis, or the expression of a paraneoplastic syndrome. The discovery of asymptomatic renal cell carcinomas has dramatically increased, since the widespread use of ultrasound and computed tomography (CT) in the study of abdominal pathology. It is worth mentioning that, in our study, although patients diagnosed with incidental tumors had a longer time free to recurrence and survival, however, the presence of clinic was not an independent risk factor for recurrence or cancer-specific survival. 
$\mathrm{C}$ ada año se diagnostican 9 millones de nuevos pacientes con cáncer y se producen 5 millones de muertos por esta enfermedad. Las neoplasias son, en los países desarrollados, la segunda causa de muerte tras las enfermedades cardiovasculares y suponen prácticamente un $10 \%$ de todas las causas de muerte. Se estima que en el año 2010 existirán 80 millones de muertos por cáncer ${ }^{1,2}$.

Los tumores del parénquima renal representan aproximadamente el 3\% de los tumores renales del adulto, siendo carcinomas más del $85 \%$ de los mismos, responsables del $2 \%$ de las muertes por cáncer $^{1}$. Más del $80 \%$ de los cánceres renales asientan en el parénquima, localizándose el resto en la pelvis renal. Prácticamente la totalidad de los tumores del parénquima son carcinomas de células renales, mientras que los tumores que asientan en la pelvis son, en su mayoría, carcinomas de células transicionales $^{3}$.

En España, en el año 2002 fallecieron 1.470 pacientes por cáncer renal, lo que constituye el 1,5\% de la mortalidad oncológica. En un estudio realizado en un área sanitaria de 90.000 habitantes entre el año 1987-2004 se calcularon las tasas de incidencia ajustada por 100.000 habitantes y año. Se evidenció una incidencia máxima de 7,21 casos por 100.000 habitantes y año. Se produjo un incremento porcentual anual durante todo el periodo de estudio de un 2,06\%, lo que incida que también en nuestro medio se está produciendo un incremento en la incidencia y es de prever que ésta se mantenga en alza en los próximos años ${ }^{4}$. Estos datos fueron corroborados a nivel internacional en otro estudio de Martin et al. ${ }^{1}$, quienes observan en la última década un aumento entre un $2,3 \%$ y un $4,3 \%^{1}$ en la incidencia de los carcinomas de células renales (CCR), debido en parte al empleo de mejores técnicas diagnósticas como la ecografía, la tomografía computerizada (TC) y la resonancia magnética (RM). Además el uso de estas en el estudio de diversas patologías abdominales, hace que hasta en un 50\% de los tumores de riñón sean diagnosticados de forma incidental antes de que se expresen clínicamente ${ }^{5}$. Aunque este fenómeno puede ser atribuido a una mejora en las técnicas de diagnóstico por imagen, que facilitarian una detección más temprana del tumor, este aumento en la incidencia no puede ser atribuido completamente a un incremento del diagnóstico de los tumores incidentales ya que tam- bién se viene observando un crecimiento de incidencia de los tumores en estadios más avanzados ${ }^{6}$. Por otro lado, diversos estudios europeos han evidenciado un aumento de la mortalidad por esta entidad $^{7,8}$

El comportamiento y la evolución del CCR son impredecibles. En ocasiones el tumor no se manifiesta clínicamente, siendo hallazgo incidental en el transcurso de estudios radiológicos por otras causas $^{9}$. Si bien su evolución es impredecible, ya que se han descrito recidivas tardías después de 10 años de la realización de la nefrectomía ${ }^{10}$ así como regresiones espontáneas del tumor ${ }^{11,12}$, el factor pronóstico más importante es el estadio tumoral en el momento del diagnóstico ${ }^{13}$, a pesar de que se han descubierto recientemente otros factores clínicos, patológicos y moleculares.

Debido al crecimiento relativamente lento cuando comienza el proceso de crecimiento y desdiferenciación del carcinoma renal, clásicamente se diagnosticaba cuando el paciente presentaba sintomas locales, sintomas derivados de las metástasis o por la expresión de un síndrome paraneoplásico ${ }^{14,15}$, motivo por el cual, también se conoce al carcinoma renal como "tumor del internista" o "el gran simulador".

El escaso conocimiento de los factores etiológicos y la desconcertante historia natural del carcinoma de células renales, hacen que la prevención primaria no sea posible. Los objetivos propuestos en nuestro estudio fueron:

- Evaluar el motivo de diagnóstico del carcinoma renal y su variación a lo largo de los años en el servicio de Urología 1, comparando los periodos 1970-1995 y 1996-2005.

- Conocer la importancia cuantitativa aproximada del diagnóstico de adenocarcinoma renal incidental en la actividad global de pruebas diagnósticas abdominales (ECO y TC) durante la práctica habitual del Servicio de Radiodiagnóstico I del HUCA.

- Determinar si el hallazgo incidental puede explicar de forma independiente el pronóstico de estos tumores o es dependiente de sus características clínico-patológicas.

\section{MATERIAL Y MÉTODOS}

Se llevó a cabo una revisión exhaustiva de las historias clínicas, de manera retrospectiva, de 373 carcinomas renales diagnosticados y sometidos a resección quirúrgica entre los años 1970 y 2005 por 
el servicio de Urología 1 del Hospital Universitario Central de Asturias. En todos los casos existió informe anatomopatológico que confirmaba la histología de carcinoma de células renales. Se descartaron, por tanto, tumoraciones benignas, (oncocitoma y angiomiolipomas), y tumoraciones malignas de otra extirpe (extremadamente raros).

De todos los casos se recogió nombre y apellidos del paciente con su número de historia, año de nacimiento y número de anatomía patológica. Se analizaron las siguientes variables:

1. Edad: menos de 50 años, entre 50-69 años, mayores o igual a 70 años.

2. Tipo histológico: células claras, papilar, cromófobas, convencional con variante de células granulares y de células fusiformes.

3. Estadio local: se utilizó la clasificación de tumores malignos TNM, de la UICC, en su sexta edición del año 2002.

4. Motivo de diagnóstico. Clínica local, clínica general sugestiva de neoplasia (síndrome constitucional, síndrome paraneoplásico), o incidental (durante el estudio de imagen por otra patología).

5. Servicio que solicitó la prueba en la que se diagnosticó el tumor.

6. Prueba con la que se realizó el diagnóstico. TC, Ultrasonidos, RM, Otra.

7. Grado nuclear de Fuhrman: se clasificaron en grado I, II, III o IV.

8. Necrosis: presencia o ausencia en la pieza.

9. Existencia o no de Invasión microvascular.

10. Tamaño tumoral en centímetros.

11. Número de tumores en la pieza de nefrectomía: único, dos, tres o múltiple.

12. Existencia de Tumor contralateral de manera simultánea.

13. Afectación ganglionar regional: $\mathrm{Nx}$ (no se pueden evaluar), NO (no se demuestran metástasis ganglionares), N1 (metástasis en un único ganglio linfático) y N2 (metástasis en más de un ganglio linfático).

14. Metástasis a distancia en el momento del diagnóstico.

15. Localización de la metástasis en el momento del diagnóstico.

16. Clínica al diagnóstico: asintomático, local (hematuria, dolor y/o masa palpable), de la metástasis, ambas (local y la de la metástasis) o síndrome general (incluida fiebre e insuficiencia renal).
17. Anemia al diagnóstico.

18. Síndrome paraneoplásico: hipercalcemia, hipergammaglobulinemia, poliglobulia, sindrome de Stauffer o tromboflebitis.

19. Tratamiento inicial: paliativo, nefrectomía radical, nefrectomía parcial o nefrectomía con trombectomía.

20. Recidiva: definida como si o no.

21. Número de recidivas: no, una, dos o más de dos.

22. Localización de la primera recidiva: local, ganglionar, torácica, hepática, difusa, ósea u otras vísceras (cerebral, cutánea, ocular, parótida, adrenal, yeyuno o vejiga).

23. Tratamiento de la primera recidiva: paliativo, exéresis, radioterapia o inmunoterapia.

24. Localización de la segunda recidiva: local, torácica, hepática, difusa, ósea u otras vísceras (cerebral, cutánea, bazo, SNC).

25. Tratamiento de la segunda recidiva: paliativo, exéresis, radioterapia o inmunoterapia.

26. Meses transcurridos hasta la primera recidiva.

27. Seguimiento total en meses.

\section{Estado actual.}

Las condiciones para considerar que un tumor era incidental eran aquellas en los que se descartó clínica local (cólico, dolor lumbar, hematuria), clínica general sugestiva de neoplasia avanzada (síndrome constitucional), síndrome paraneoplásico o búsqueda de una neoplasia oculta. Dado que en los últimos años se ha incrementado el número de tumores incidentales comparamos los últimos 10 años con los anteriores.

Se excluyeron del estudio los pacientes que:

- No tenían historia clínica completa.

- Tenían tumor de riñón no carcinoma.

El análisis estadístico se realizó mediante el programa SPSS, versión 11. Se consideró la existencia de significación estadística cuando la $p$ fue menor de 0,05. Se recogieron variables de cada tumor referidas a las características de la neoplasia y a la evolución de los pacientes en cuanto a tiempo libre de enfermedad y mortalidad. Fueron 25 variables recogidas de cada tumor primario o de cada recidiva.

Las variables cuantitativas se expresan como media +/- DE (desviación estándar) o bien como mediana (percentil 50) en los casos pertinentes (tiempo de supervivencia). Se utilizó el test de Student para comparar las medias de dos categorías dentro 
de una misma variable dependiente y el Análisis de la Varianza ANOVA (razón F) para comparar más de dos medias. Algunas de las variables cuantitativas fueron categorizadas para un análisis ulterior más sencillo. Las variables cualitativas se expresaron como frecuencias y porcentajes. Se realizaron agrupaciones de variables cualitativas en conjuntos homogéneos para facilitar el estudio multivariante posterior. Para conocer la existencia de asociación entre variables se llevaron a cabo tablas de contingencia y la prueba de Chi2 de Pearson. Se calculó la significación para este estadístico.

El análisis de supervivencia (tiempo libre de enfermedad y de supervivencia libre de cáncer) se realizó mediante el método de Kaplan-Maier, lo cual permitió su representación gráfica. Posteriormente, se realizó la prueba del rango logarítmico (lon-rank), comparando de forma univariante las curvas de supervivencia y evaluando así el efecto de cada uno de los factores pronósticos sobre los tiempos libres de recurrencia y progresión. Por último, consideramos las variables que fueron significativas en el análisis univariante como covariantes en la prueba del modelo de riesgos proporcionales de Cox, con la intención de determinar el efecto conjunto de los factores pronósticos que resultan significativos de forma independiente y cuantificar los riesgos relativos (Hazard Ratio) e intervalos de confianza (IC) del 95\% de recurrencia y supervivencia. Se eligió el procedimiento por pasos hacia delante, determinando el estadístico de Wald, que introduce y selecciona variables en un orden que depende del porcentaje de la varianza.

\section{RESULTADOS}

\section{Descripción de la serie}

Los casos elegidos comprendieron a pacientes con una edad media al diagnóstico de 61,77 años \pm 12,02 años (16-88). En un 89,5\% de pacientes, ( $n=334)$ la estirpe tisular fue de células claras, seguido del papilar $(n=12)$, y la variante con células granulares $(n=8)$, con muy pocos casos correspondientes a un patrón con células fusiformes, $(n=7)$, o con células cromófobas u otros $(n=12)$. En 199 casos el tumor afectaba al riñón derecho $(53,4 \%)$, en 170 casos al izquierdo (45,6\%), mientras que en 4 casos el tumor era bilateral $(1,1 \%)$.

De los 373 tumores de nuestra serie, 146 $(39,1 \%)$ fueron diagnosticados según nuestros crite- rios de forma incidental, mientras que 227 (60,9\%) lo fueron por presentar clínica sospechosa de presencia de un tumor. Cuando realizamos el recuento distribuyendo estos pacientes en dos grupos, por un lado los diagnosticados entre los años 1970-1995, y por otro lado los diagnosticados entre 1996-2005, observamos un incremento significativo en el número de tumores diagnosticados de forma incidental en el segundo periodo, ya que en el periodo 1970-1995, tan sólo un $24,9 \%$ de los tumores se diagnosticaron de forma incidental (48/193), frente al 54,4\% de los diagnosticados entre 1996-2005 (98/180).

Respecto a los servicios implicados en el diagnóstico incidental de estos tumores se encuentran por orden de frecuencia, Urología (48 pacientes diagnosticados), Médicos de Atención Primaria (30), Nefrología y Digestivo (16 pacientes cada uno), Cirugía general (8), Medicina Interna (5), Endocrino (4), Ginecología (4), Neumología (4), Cirugía vascular (3), Neurología (3), Hematología (2), Reumatología (1), UVI (1) y Traumatología (1) (Fig. 1). Entre las causas por las que se solicitaron estos estudios de imagen fueron las siguientes las más frecuentes: En el servicio de Urología en 32 casos fueron estudios por prostatismo, en Nefrología en 14 casos el estudio de imagen se solicitó en controles de pacientes hipertensos, siendo en Cirugía, Digestivo y Medicina Interna, trastornos gastrointestinales con o sin dolor abdominal (dispepsia, diarrea, dolor...) el motivo de diagnóstico en 26 pacientes.

Respecto a los estudios de imagen solicitados durante los periodos estudiados, realizamos una comparativa entre los últimos años de cada periodo, es decir, entre el año 1995 y el 2005. Observamos como era de esperar un incremento muy significativo

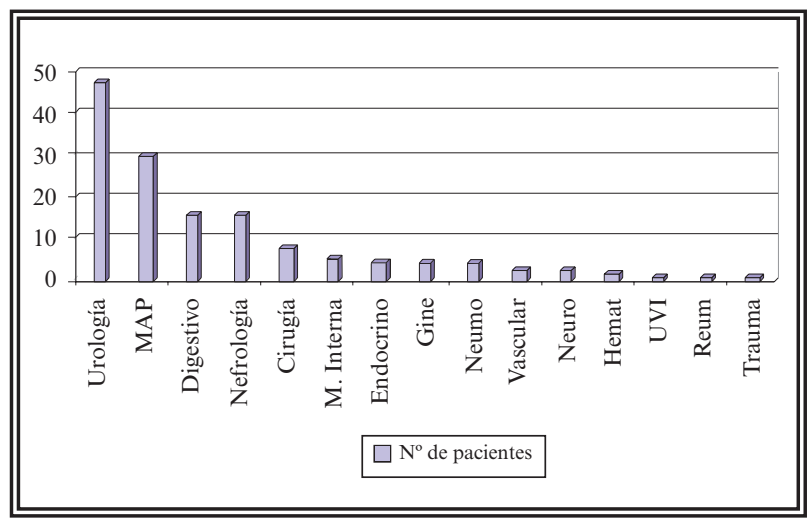

FIGURA 1. Servicios implicados en el diagnóstico de tumores renales incidentales. 
en el año 2005 respecto al número de ecografías y TC realizados en el HUCA, de tal manera que en 1995 se realizaron un total de 5973 ecografias frente a las 14.831 realizadas en 2005 , lo que supuso un incremento del 148,3\%. En los TC realizados ocurrió lo mismo, con una diferencia que también resultó manifiesta, ya que se pasaron de 6.328 pruebas realizadas en 1995 a 12.167 en el año 2005, lo que supuso un incremento del 92,27\% (Fig. 2).

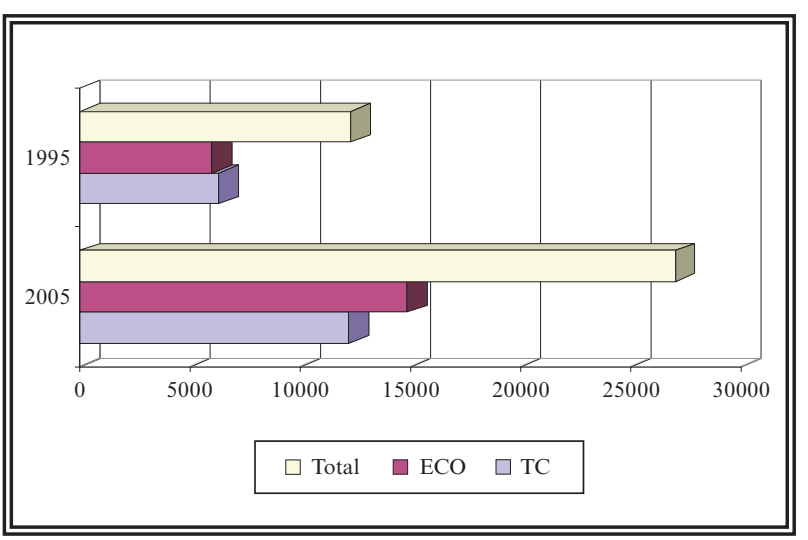

FIGURA 2. Número de pruebas de imagen solicitadas en los años 1995 y 2005.

Observamos, como era de esperar, un incremento de solicitudes de pruebas de imágenes en todos los servicios, (datos resumidos en las Figs. 3 y 4). En concreto en el servicio de Urología 1 se solicitaron un total de 674 ecografías en el año 1995, frente a las 1646 solicitadas en 2005. Lo mismo ocurrió en cuanto al número de TC solicitados, si bien la diferencia no fue tan significativa, ya que en 1995 se solicitaron 231, frente a los 336 solicitados en el año 2005. En el resto de servicios las diferencias fueron también significativas como muestran las figuras, guardando clara relación el incremento de tumores renales diagnosticados de forma incidental con el aumento del número de pruebas realizadas.

Respecto al estadio tumoral en el momento del diagnóstico, en el grupo de tumores incidentales, 97/143 fueron pT1, mientras que en el grupo de tumores sintomáticos, 91/220 casos eran pT3. Este dato se pudo calcular en 363 de los 373 pacientes, ya que en 10 casos no pudo determinarse. Además, el $84,5 \%$ de los tumores incidentales (n:116), eran tumores G1-G2, mientras que el 64,7\% de los tumores con sintomatología (n:130), eran tumores de alto grado G3-G4. Este dato pudo determinarse en 311 pacientes.

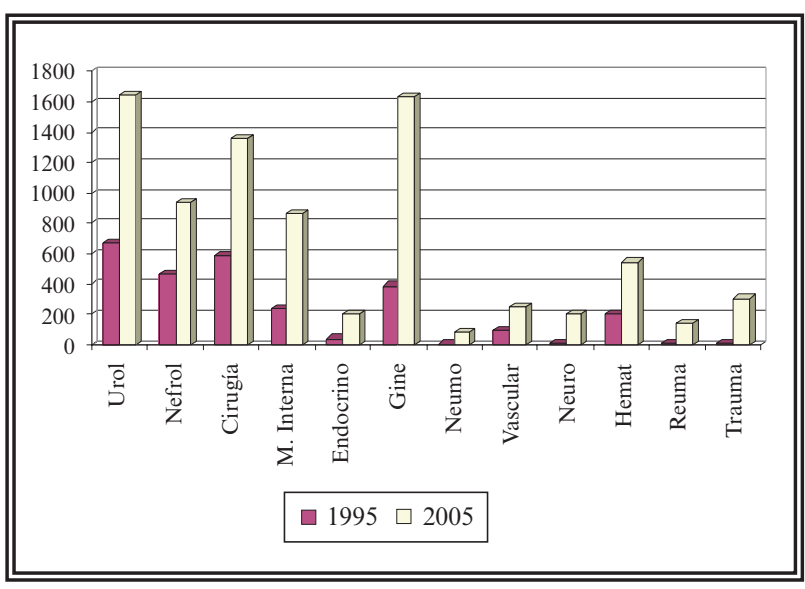

FIGURA 3. Número de Ecografias solicitados por servicio y año.

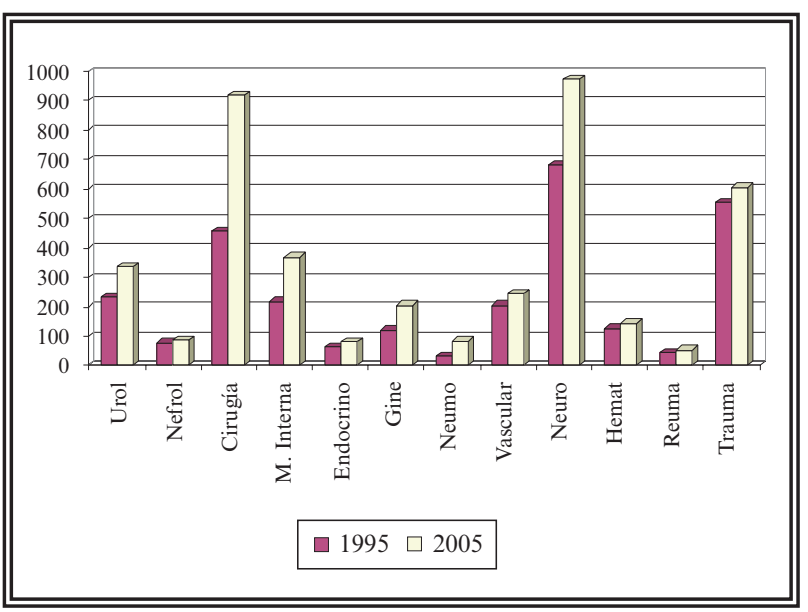

FIGURA 4. Número de TC solicitados por servicio y año.

En cuanto a las características anatomopatológicas, observamos ausencia de necrosis en el 68,1\% (n:98) de los tumores incidentales, mientras que se evidenció necrosis en el 54,6\% de los tumores sintomáticos (n:118). Asímismo, no existió invasión microvascular de la pieza quirúrgica en hasta 133 pacientes (93,0\%) del grupo de tumores incidentales frente al 27,8\% (n:60) del grupo de tumores sintomáticos que presentaron invasión microvascular de la pieza quirúrgica.

Estudiamos también la presencia de anemia en el momento de diagnóstico, ya que este factor parece relacionarse con un peor pronóstico, encontrando ausencia de la misma en el 88,4\% (n:129) en el grupo de pacientes diagnosticados de forma incidental, mientras que en el grupo de pacientes con tumores sintomáticos, hasta el 31,1\% (n:71), cursaron con anemia en el momento del diagnóstico. 
Cuando estudiamos la implicación del diagnóstico incidental en la tasa de recidivas del tumor y en la supervivencia global del cáncer renal, encontramos respecto a la tasa de supervivencia, o tiempo libre de enfermedad, como los pacientes con sintomas en el momento del diagnóstico, un 32,9\% (n:75) presentaron recidivas en el transcurso del seguimiento, frente a sólo el 15,8\% de los pacientes con tumores incidentales (n:23) (Fig. 5).

Respecto a la supervivencia cáncer específica, observamos en nuestro estudio diferencias significativas entre el grupo de pacientes con tumores incidentales y aquellos sintomáticos ( $\mathrm{p}=0,0013)$ (Fig. 6).

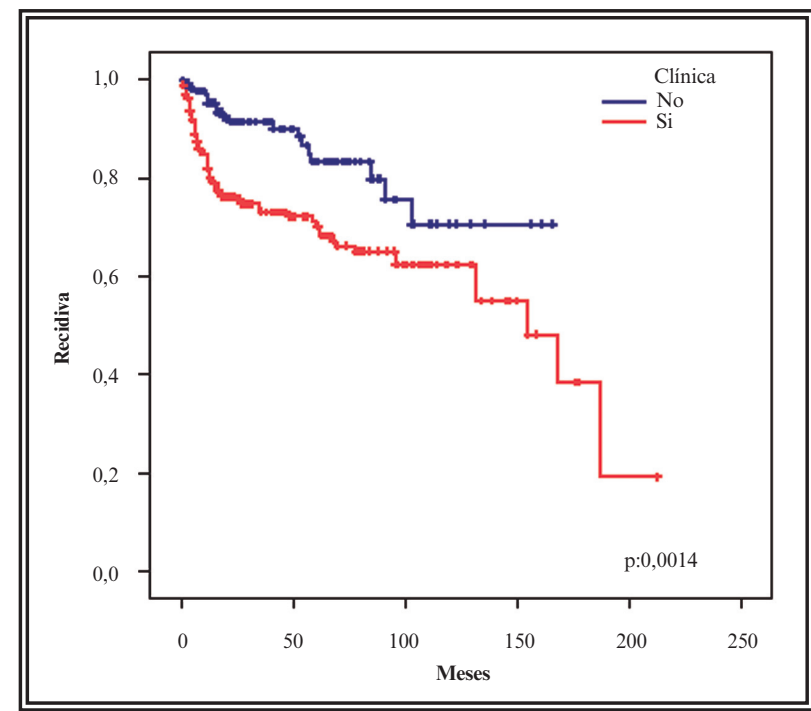

FIGURA 5. Supervivencia libre de enfermedad durante el seguimiento.

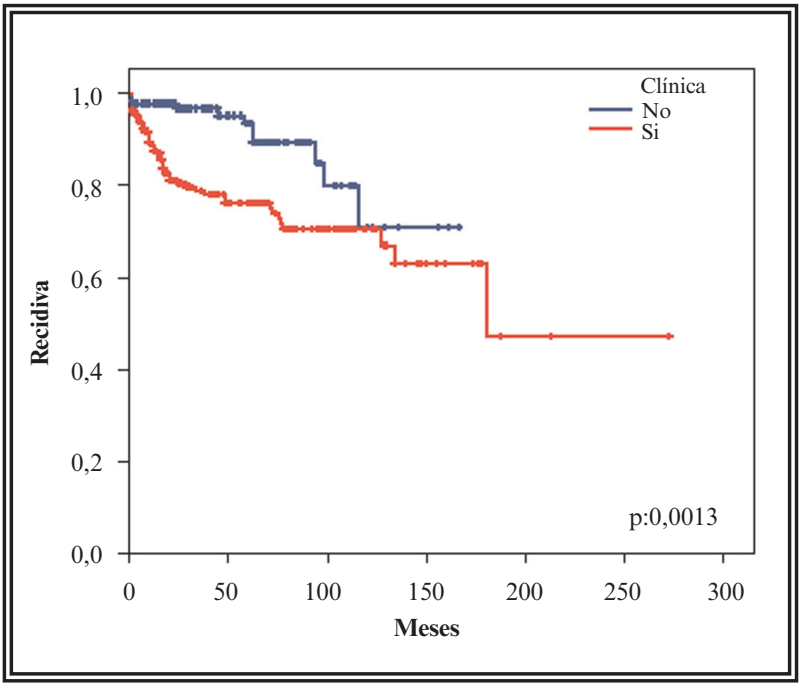

FIGURA 6. Supervivencia cáncer específica en ambos grupos.

\section{Asociaciones univariantes y la comparación de medias}

Se encontraron varias asociaciones significativas (prueba de $\mathrm{Chi}^{2}$ ), destacando:

- Los pacientes con tumores incidentales tenían al diagnóstico una edad media significativamente mayor $(\mathrm{p}=0,003)$.

- Los pacientes con tumores sintomáticos, presentaron una mayor proporción de tumores T2 y sobre todo T3-4 en el momento del diagnóstico, mientras que en los pacientes con tumores incidentales predominaron neoplasias intrarrenales resultando esta diferencia ser significativamente estadística $(\mathrm{p}<0,0001)$.

- Encontramos una mayor proporción de tumores de alto grado (G3-G4) entre los pacientes que presentaban clínica y una menor proporción de estos en tumores incidentales, en los cuales predominaron los tumores de grado bajo o intermedio (G1-G2), resultando el valor de $\mathrm{p}=0,0011$.

- Se evidenció presencia de necrosis en la pieza tumoral, infiltración vascular microscópica, mayor de lo esperado entre los tumores que presentaron clínica $(\mathrm{p}<0,0001)$.

- Los pacientes con tumores sintomáticos en el momento de diagnóstico, presentaron significativamente un mayor porcentaje de anemia $(\mathrm{p}<0,0001)$.

- Observamos presencia de adenopatías y metástasis a distancia en el momento del diagnóstico, siendo mayor de lo esperado entre los tumores que presentaron clínica y menor en tumores incidentales $(\mathrm{p}<0,0001)$.

\section{Estudio de supervivencia libre de enfermedad y supervivencia cáncer específica}

Los factores que demostraron significación estadística en el tiempo de supervivencia relacionada con el carcinoma renal (prueba de log rank) en nuestra serie fueron los siguientes:

- Los tumores sintomáticos al diagnóstico, presentaron una mayor tasa de recidivas, siendo esta significativamente estadística $(\mathrm{p}<0,0001)$.

- Los tumores incidentales en el diagnóstico, tuvieron una supervivencia cáncer específica mayor que el grupo de pacientes con tumores sintomáti$\cos (\mathrm{p}=0,0013)$. 
Factores independientes implicados en la supervivencia libre de enfermedad

Se realizó un estudio multivariante, calculándose las "odds ratio" (OR) para cada factor, incluyéndose también los valores del índice de confianza del 95\% de la OR. De los factores estudiados (Grado nuclear, tamaño tumoral, clasificación TNM, infiltración vascular, presencia de necrosis en la pieza quirúrgica, anemia en el momento de diagnóstico y la presencia de sintomatología en el momento de diagnóstico), los que se comportaron como factores independientes implicados en la recidiva del tumor resultaron ser los siguientes:

- Presencia de T3 (OR: 4,325; IC 95\%: 1,212-15,434).

- Grado Nuclear (OR: 1,617; IC 95\%: 1,114-2,347).

- N+: presencia de adenopatías metastásicas (OR: 3,817; IC 95\%: 1,568-9,573).

- Presencia de Anemia (OR: 2,590: IC 95\%; 1,3844,846).

\section{Factores independientes implicados en la supervivencia cáncer especifica:}

Se realizó igualmente un estudio multivariante, calculándose de nuevo las Odds Ratio para cada factor. De entre los factores estudiados (Grado nuclear, tamaño tumoral, clasificación TNM, infiltración vascular y necrosis de la pieza quirúrgica, presencia de anemia, sintomatología en el diagnóstico, tipo de recidiva y tratamiento de la recidiva (quirúrgico vs otros)), encontramos como factores independientes en la supervivencia cáncer específica los siguientes.

- Invasión microvascular (OR: 2,090: IC 95\%; 1,019-4,287).

- M+: presencia de metástasis (OR: 3,280: IC 95\%; 1,459-7,373).

- Tto. recidiva (quirúrgico vs otros) (OR: 1,298: IC 95\%; 1,209-1,395).

\section{DISCUSIÓN}

El tumor maligno del riñón o carcinoma de células renales, denominación en uso en la actualidad, es relativamente frecuente y constituye el 3\% del total de tumores en el hombre y el $85 \%$ de todos los tumores renales. Desde el punto de vista clínico el tumor renal se encuentra entre las neoplasias humanas de evolución menos predecible, pudiendo permanecer el paciente completamente asintomático con una tumoración voluminosa y sin embargo, en otras ocasiones es desde su diagnostico, agresivo y destructor. Bassil B y Dosoretz $\mathrm{DE}^{16}$, describen ya en 1985 que el 30\% de los pacientes con este tumor presentan un crecimiento del mismo y manifestaciones clínicas que pueden ser variadas y hasta un $30 \%$ de los portadores de este estado patológico se diagnostican por síntomas y signos no urológicos por lo que en algunas ocasiones esta situación pone en tela de juicio la agudeza diagnostica del medico por lo que en el diagnostico de esta entidad clínica, en la actualidad, cobran gran valor el uso de métodos de imagen, para un diagnóstico precoz del mismo.

En nuestro estudio, observamos claras diferencias anatomopatológicas en los tumores entre ambas series, destacando una mayor proporción de tumores localizados pT1a-pT1b $(67,9 \%)$ en el grupo de tumores incidentales, frente a una mayor proporción de tumores extrarrenales pT3-pT4 (41,4\%) en el grupo de tumores sintomáticos. Asímismo, observamos que entre el grupo de tumores asintomáticos, había una mayor proporción de tumores de bajo grado nuclear (G1-G2) 64,7\% frente a una mayor proporción de G3-G4, (25,2\%), en tumores sintomáticos. Clásicamente el adenocarcinoma renal se diagnosticaba cuando presentaba sintomas locales (la hematuria y el dolor lumbar, eran los más frecuentes), síntomas derivados de las metástasis o por la expresión de un síndrome paraneoplásico. Desde la generalización de la ecografía y la tomografía computerizada (TC) en el estudio de la patología abdominal ha aumentado de forma espectacular el descubrimiento de adenocarcinomas renales asintomáticos. Encontramos de nuevo diferencias significativas al estudiar la presencia de adenopatías y metástasis a distancia $(\mathrm{p}<0,0001)$, en el momento del diagnóstico, siendo mayor de lo esperado entre los tumores que presentaron clínica y menor en tumores incidentales, $(12,7 \%$ frente a $1,4 \%)$. Jalón et al. ${ }^{13}$, en un estudio previo realizado, encontraron estos factores como independientes de mortalidad cáncer específica en estos tumores. Otro factores microscópicos analizados en la pieza quirúrgica fueron la presencia de necrosis y la invasión microvascular, encontrando también una mayor proporción de tumores sin ninguna de estas características en el grupo de incidentales $(68,1 \%$ y $93,0 \%$ ), así como una mayor proporción de tumores con estas características en el grupo de tumores 
sintomáticos (54,6\% y 27,8\%). Como cabía esperar por los hallazgos clínico-patológicos en ambos grupos, observamos una mayor tasa de recidivas en pacientes sintomáticos (32,9\% frente al 15,8\%), así como una menor tasa de supervivencia.

De los 373 tumores de nuestra serie, 146 $(39,1 \%)$ fueron diagnosticados según nuestros criterios de forma incidental, mientras que 227 (60,9\%) lo fueron por presentar clínica sospechosa de presencia de un tumor. Cuando realizamos el recuento distribuyendo estos pacientes en dos grupos, por un lado los diagnosticados entre los años 1970-1995, y por otro lado los diagnosticados entre 1996-2005, observamos un incremento significativo en el número de tumores diagnosticados de forma incidental en el segundo periodo, ya que en el periodo 1970-1995, tan sólo un $24,9 \%$ de los tumores se diagnosticaron de forma incidental, frente al 54,4\% de los diagnosticados entre 1996-2005. Observamos, como era de esperar, un incremento de solicitudes de pruebas de imágenes en todos los servicios. En concreto en el servicio de Urología 1 se solicitaron un total de 674 ecografías en el año 1995, frente a las 1646 solicitadas en 2005. Lo mismo ocurrió en cuanto al número de TC solicitados, si bien la diferencia no fue tan significativa, ya que en 1995 se solicitaron 231 , frente a los 336 solicitados en el año 2005. En el resto de servicios las diferencias fueron también significativas como muestran las figuras, guardando clara relación el incremento de tumores renales diagnosticados de forma incidental con el aumento del número de pruebas diagnósticas.

En el estudio univariante de los factores implicados en el tiempo libre de enfermedad, el aumento del tamaño tumoral disminuía significativamente la supervivencia, sin haber diferencias significativas en la supervivencia entre pTla y pT1b. De 116 casos de CCR con tamaño tumoral recogido en la serie de Delahunt et al. ${ }^{17}$, encuentran que en el análisis univariante sólo el grado Fuhrman y el score AgNOR estaban asociados con la supervivencia y, sin embargo, en el análisis multivariante sólo el tamaño tumoral se mantuvo significativo. Concluyen que aunque el punto de corte de la clasificación TNM en pT1 y pT2, y el propósito de subdividir el estadio pT1 se correlacionan con la supervivencia, el tamaño como parámetro pronóstico del CCR es relativo más que indicativo al ser una variable continua.
Salama et al. ${ }^{18}$ estudian el impacto del tamaño tumoral sobre la supervivencia cáncer específica y la recurrencia, en una serie de 259 pacientes con tumores renales. Proponen que subestratificar el CCR en pTla y pTlb produce información pronóstica importante, validando las modificaciones del TNM del 2002. El punto de corte de 4.0 y $7.0 \mathrm{~cm}$ es razonable y práctico para tumores pTla-T1b y pT1b-T2. Así mismo, concluyen que la subestratificación de pT1 es particularmente útil en tumores con histología de CCR y que no parece justificada ninguna modificación de los tumores pT1 y pT2.

\section{CONCLUSIONES}

- Los pacientes diagnosticados con tumores incidentales presentaron un mayor tiempo libre de enfermedad y de supervivencia.

- La presencia de metástasis, la invasión microvascular de la pieza quirúrgica y el tratamiento de la recidiva (quirúrgico vs otros), resultaron ser factores independientes en la supervivencia cáncer específica.

- El grado nuclear, la presencia de adenopatías y el estadio T3, actuaron como factores independientes de la recidiva.

- La presencia de clínica no fue un factor independiente de recidiva ni de supervivencia cáncer específico.

- Las diferencias en el tiempo libre y supervivencia dependerían de otras características clínico-patológicas previamente descritas que son diferentes en los tumores incidentales frente a aquellos que presentan clínica.

\section{REFERENCIAS}

1. Martin Jerry L.Cancer Epidemiology. In Super course Lectures. En: http://www.pitt.edu/super1/lectura/lec0192/001.htm.

2. Parkin CM, Whelan SL, Ferlay J. Cancer incidence en five continents, volume VIII. IARC Scientific Publications No. 155. Lyon, France, International Agency for research on Cancer, 2002.

3. Jayson M, Saunders H. Increased incidence of serendipitously discovered renal cell carcinoma. Urology. 1998;51(2):203-205.

4. Santos Arrontes D, de Castro Barbosa F, Valer López-Fando MP, Fernández Arjona M, Cortés Aránguez I. Carcinoma renal de células claras. Análisis de incidencia y supervivencia en un área sanitaria de 90000 habitantes. Arch Esp Urol. 2006;59(3):233-237.

4. Santos Arrontes D, de Castro Barbosa F, Valer López-Fando MP, Fernández Arjona M, Cortés Aránguez I. Renal cell renal carcinoma. Analysis of incidence and survival in a 90.000 citizens healthcare area. Arch Esp Urol. 2006;59(3):233-237.

5. Pretorius ES, Sielgeman ES, Ramchandani P, Cangiano T, Banner MP. Renal neoplasms amenable to partial nephrectomy: MR imaging. Radiology 1999; 212: 28-34. 
6. Mathew A, Devesa SS, Fraumeni JF Jr. Global increases in kidney cancer incidence, 1973-1992. Eur J Cancer Prev. 2002;11: 171-178.

7. La Vecchia C, Levi F, Lucchini F, Negri E. Descriptive epidemiology of kidney cancer in Europe. J Nephrol 1992; 5: 37-43.

8. Aso Y, Homma Y, A suvey on incidental renal cell carcinoma in Japan. J Urol. 1992;147:340-347.

9. How W-H, Devesa SS, Warren JL, Fraumeni JF Jr. Rising incidence of renal cell carcinoma in the United States. JAMA. 1999;281:1628-1631.

10. Glaspy JA. Therapeutic options in the management of renal cell carcinoma. Semin Oncol. 2002;29(3 Suppl 7):41-46.

11. De Riese W, Goldenberg K, Allhoff E, Stief C, Schlick R, Liedke $\mathrm{S}$, et al. Metastatic renal cell carcinoma ( $\mathrm{RCC}$ ): spontaneous regression, long-term survival and late recurrence. Int Urol Nephrol. 1991;23(1):13-25.

12. Jalón Monzón A, Regadera Sejas FJ, García J, Martínez F, Sánchez T, Fernández Gómez J, Rodríguez JJ, González Álvarez RC, Álvarez-Múgica M. Regresión espontanea de trombosis venosa en un caso de carcinoma renal. Arch Esp Urol. 2005;58(3):50-53.

13. Jalón Monzón A, Álvarez Múgica M, Fernández Gómez JM, Martín Benito JL, Martínez Gómez F, García Rodríguez J, et al. Adenocarcinoma de células renales: factores pronósticos y estadificación. Arch Esp Urol. 2007;60(2):125-136.
14. McNicholls DW, Segura JW, DeWeerd JH. Renal cell carcinoma: long-term survival and late recurrence. J Urol. 1981:126(1):17-23.

15. Middleton RG. Surgery for metastatic renal cell carcinoma. J Urol. 1967;97(6):973-980.

16. Bassil B, Dosoretz DE, Prout GR. Validation of tumor, nodes, metastasis classification of renal cell carcinoma. J Urol. 1985; 134(3):450-454.

17. Delahunt B, Kittelson JM, McCredie MR, Reeve AE, Stewart JH, Bilous AM. Prognostic importance of tumor size for localized conventional (clear cell) renal cell carcinoma. Assessment of TNM T1 and T2 tumor categories and comparison with other prognostic parameters. Cancer. 2002;94(3):658-667.

18. Salama ME, Guru K, Stricker H, Peterson E, Peabody J, Menon $\mathrm{M}$, et al. pT1 substaging in renal cell carcinoma: validation of the 2002 TNM staging modification of malignant tenal epithelial tumors. J Urol. 2005;173(5):1492-2005.

Correspondencia autor: Dra. V. Bulnes Vázquez

Servicio de Urología 1. Hospital Universitario Central de Asturias Celestino Villamil, s/n - 33006 Oviedo (Asturias)

Tel.: 985108004

E-mail autor: vebulnesv@yahoo.es

Información artículo: Original - Cáncer renal

Trabajo recibido: enero 2008

Trabajo aceptado: junio 2008 